To appear in ApJ-Letters; preprint June 17, 2002

\title{
A dynamical mechanism for establishing apsidal resonance
}

\author{
Renu Malhotra \\ Department of Planetary Sciences, University of Arizona, 1629 E. University Blvd., Tucson, AZ \\ 85721,renu@lpl.arizona.edu
}

\begin{abstract}
We show that in a system of two planets initially in nearly circular orbits, an impulse perturbation that imparts a finite eccentricity to one planet's orbit causes the other planet's orbit to become eccentric as well, and also naturally results in a libration of their relative apsidal longitudes for a wide range of initial conditions. We suggest that such a mechanism may explain orbital eccentricities and apsidal resonance in some exo-planetary systems. The eccentricity impulse could be caused by the ejection of a planet from these systems, or by torques from a primordial gas disk. The amplitude of secular variations provides an observational constraint on the dynamical history of such systems.
\end{abstract}

Subject headings: planetary systems — celestial mechanics — instabilities - stars:individual $(v$ Andromedae, HD 83443)

\section{Introduction}

Orbital eccentricities in exo-planetary systems ${ }^{1}$ discovered thus far are often surprisingly large and have proven to be a major puzzle in understanding these systems. At least two systems with multiple planets in eccentric orbits ( $v$ Andromedae and HD 83443) are suspected of exhibiting secular apsidal resonance (Chiang, Tabachnik \& Tremaine 2001, Wu \& Goldreich 2002). Apsidal resonance is the phenomenon of phase-locking of the apsidal longitudes of two orbits, such that the two planets have a common average rate of apsidal precession and the angular difference of their apsidal longitudes, $\Delta \varpi=\varpi_{1}-\varpi_{2}$, librates around 0 .

We describe here a dynamical mechanism for establishing apsidal resonance in a pair of planets that are initially on nearly circular orbits. We use classical analysis from celestial mechanics to show that an impulse perturbation that imparts an eccentricity to one of the orbits, excites the other planet's eccentricity on a secular timescale and also results in the libration of the relative apsidal

\footnotetext{
${ }^{1}$ http://www.exoplanets.org
} 
longitude for a wide range of initial conditions. A plausible cause of an impulse perturbation is the ejection of a planet from the system; torques from an exterior primordial disk may also cause such an eccentricity impulse.

\section{Secular dynamics}

Consider a pair of planets in well-separated orbits, whose orbital period ratio is not too close to a ratio of two small integers. Under the assumption that the relative inclination of their orbital planes is small, the secular interactions in such a system preserve the orbital semimajor axes and induce quasi-periodic variations in the other orbital parameters. For small eccentricities and small relative inclinations, the eccentricity-apsidal line variations are decoupled from the inclinationnodal line variations (Murray \& Dermott 1999). Under these conditions, the secular variations of the eccentricities and apsidal longitudes are described by a set of first order linear differential equations for the components of the so-called eccentricity vectors, $\left(k_{j}(t), h_{j}(t)\right)=e_{j}\left(\cos \varpi_{j}, \sin \varpi_{j}\right)$, for each of the planets, where $e_{j}$ is the eccentricity and $\varpi_{j}$ is the apsidal longitude. The general solution for two planets is a superposition of two eigenmodes,

$$
\begin{aligned}
h_{j}(t) & =E_{j}^{(1)} \sin \left(g_{1} t+\beta_{1}\right)+E_{j}^{(2)} \sin \left(g_{2} t+\beta_{2}\right), \\
k_{j}(t) & =E_{j}^{(1)} \cos \left(g_{1} t+\beta_{1}\right)+E_{j}^{(2)} \cos \left(g_{2} t+\beta_{2}\right) .
\end{aligned}
$$

Let $m_{\star}, m_{1}, m_{2}$ be the masses of the star, the inner planet and the outer planet, respectively; $n_{1}$, $n_{2}$ the orbital mean motions of the inner and outer planet, respectively; $\alpha=a_{1} / a_{2}<1$ the ratio of the semimajor axes, $b_{s}^{(l)}(\alpha)$ a Laplace coefficient. We also define $\mu=m_{1} / m_{2}$, and $B=b_{3 / 2}^{(2)} / b_{3 / 2}^{(1)}$. With these definitions, the mode frequencies are

$$
g_{1,2}=\frac{1}{8} \sqrt{\alpha} b_{3 / 2}^{(1)}(\alpha) \frac{m_{2}}{m_{\star}} n_{2}\left\{1+\mu \sqrt{\alpha} \pm\left[(1-\mu \sqrt{\alpha})^{2}+4 \mu \sqrt{\alpha} B^{2}\right]^{1 / 2}\right\}
$$

and the eigenvector amplitudes $\mathbf{E}^{(j)}$ are given, up to a normalization factor, by

$$
\frac{E_{2}^{(j)}}{E_{1}^{(j)}}=\frac{1-\mu \sqrt{\alpha} \mp\left[(1-\mu \sqrt{\alpha})^{2}+4 \mu \sqrt{\alpha} B^{2}\right]^{1 / 2}}{2 B} \equiv \rho_{j},
$$

where the ' - ' $\operatorname{sign}$ in $\mp$ corresponds to $j=1$, and the ' $+{ }^{\prime}$ sign to $j=2$. The phases $\beta_{j}$ and the normalization factors for the amplitudes of the modes are determined by initial conditions.

We assume initially nearly circular orbits, as is expected of planet formation in a dissipative protoplanetary disk. And we consider an impulse perturbation ${ }^{2}$ that imparts a finite eccentricity,

\footnotetext{
${ }^{2}$ In general, an impulse perturbation would result in not only an eccentricity impulse but also impulse perturbation to other orbital elements. The secular dynamics described here remains valid provided we use the post-impulse value of $a_{2}$ in evaluating $\alpha$ and $n_{2}$.
} 
$e_{2 \mathrm{f}}$, to the outer planet's orbit, at time $t=0$. (The case of an impulse to the inner planet's orbit is solved in a similar way.) Then, with initial conditions $h_{1}(0)=h_{10}, k_{1}(0)=k_{10}, h_{2}(0)=0, k_{2}(0)=$ $e_{2 \mathrm{f}}$, we have

$$
\begin{aligned}
& E_{1}^{(1)} \cos \beta_{1}=\frac{\rho_{2} k_{10}-e_{2 \mathrm{f}}}{\rho_{2}-\rho_{1}}, \quad E_{1}^{(1)} \sin \beta_{1}=\frac{\rho_{2} h_{10}}{\rho_{2}-\rho_{1}}, \\
& E_{1}^{(2)} \cos \beta_{2}=\frac{\rho_{1} k_{10}-e_{2 f}}{\rho_{1}-\rho_{2}}, \quad E_{1}^{(2)} \sin \beta_{2}=\frac{\rho_{1} h_{10}}{\rho_{1}-\rho_{2}} \text {. }
\end{aligned}
$$

This solution admits either circulation or libration of the relative apsidal longitude, $\Delta \varpi$. Examples of three types of possible behavior are shown in Fig. 1. In each of these cases, the cumulative probability density function of $|\Delta \varpi|$ and of $e_{1}$ (i.e., the fraction of time that $|\Delta \varpi|, e_{1}$ is less than some value) is also shown.

In the case of zero initial eccentricity, $e_{10}=0$ [Fig. $1(\mathrm{a}-\mathrm{d})$ ], mode 1 and mode 2 appear with equal amplitude but opposite phase in the inner planet's eccentricity vector. The time evolution of the planets' eccentricities and of $\Delta \varpi$ is given by

$$
\begin{aligned}
e_{1}(t) & =\frac{\sqrt{2} e_{2 \mathrm{f}}}{\left|\rho_{1}-\rho_{2}\right|}\left[1-\cos \left(g_{1}-g_{2}\right) t\right]^{1 / 2}, \\
e_{2}(t) & =\frac{e_{2 \mathrm{f}}}{\left|\rho_{1}-\rho_{2}\right|}\left[\rho_{1}^{2}+\rho_{2}^{2}-2 \rho_{1} \rho_{2} \cos \left(g_{1}-g_{2}\right) t\right]^{1 / 2}, \\
\tan \Delta \varpi(t) & =\frac{\rho_{2}-\rho_{1}}{\rho_{2}+\rho_{1}} \tan \left[\frac{1}{2}\left(g_{1}-g_{2}\right) t+\frac{\pi}{2}\right] .
\end{aligned}
$$

The eccentricity of the inner planet grows from its initial value of zero to a value comparable to $e_{2 \mathrm{f}}$ on the secular timescale $\sim\left|g_{1}-g_{2}\right|^{-1}$; the eccentricities vary periodically (but not sinusoidally) with a common frequency, $g_{1}-g_{2}$, and with opposite phases. The inner planet's eccentricity has a maximum excursion to $2 e_{2 f} /\left|\rho_{1}-\rho_{2}\right|$, while the eccentricity of the outer planet varies between a minimum of $e_{2 \mathrm{f}}\left|\rho_{1}+\rho_{2}\right| /\left|\rho_{1}-\rho_{2}\right|$ and a maximum of $e_{2 \mathrm{f}}$. The apsidal longitude difference is limited to $\left(-90^{\circ}, 90^{\circ}\right)$; it oscillates with the same frequency, $g_{1}-g_{2}$. The singularity of the tangent function at $\pm \frac{1}{2} \pi$ implies a discontinuity in the time evolution of $\Delta \varpi$. This discontinuity is not unphysical (see Fig. 2 below): as the eccentricity vector of the inner planet passes periodically through the origin, its apsidal longitude changes discontinuously.

A small non-zero initial eccentricity, $e_{10} \ll e_{2 f}$, results in apsidal libration with an amplitude smaller than $90^{\circ}$ for values of $\varpi_{10}$ that are approximately within $\pm 90^{\circ}$ of $\varpi_{20}$ [e.g., Fig. $1(\mathrm{e}-\mathrm{h})$ ], and apsidal circulation otherwise [e.g., Fig. 1(i-l)]. In all cases, the secular dynamics following an impulse perturbation to $e_{2}$ yields a low probability of finding $|\Delta \varpi|>90^{\circ}$ [see Fig. 1(c,g,k)], and only $\sim 1 / 3$ probability of finding $e_{1}$ less than half its maximum [see Fig. 1(d,h,l)]. As we show below, a small non-zero $e_{10}$ results in apsidal resonance with approximately $50 \%$ probability.

It is illustrative to consider the limiting case $m_{1} \longrightarrow 0$. In this case, the orbital elements of the outer planet are constant: $k_{2}(t)=e_{2 \mathrm{f}}, h_{2}(t)=0$; for the inner planet, we have the following solution:

$$
k_{1}(t)=+\left(k_{10}-e_{1 \mathrm{f}}\right) \cos g t+h_{10} \sin g t+e_{1 \mathrm{f}},
$$




$$
h_{1}(t)=-\left(k_{10}-e_{1 \mathrm{f}}\right) \sin g t+h_{10} \cos g t,
$$

with

$$
e_{1 \mathrm{f}}=B e_{2 \mathrm{f}} \quad \text { and } \quad g=\frac{1}{4} n_{1} \frac{m_{2}}{m_{\star}} \alpha^{2} b_{3 / 2}^{(1)}(\alpha) .
$$

This solution admits a simple geometrical interpretation as illustrated in Fig. 2a: the inner planet's eccentricity is the vector sum of a 'forced' component of magnitude $e_{1 \mathrm{f}}$ which is aligned with the outer planet's apsidal longitude (taken to be zero here, without loss of generality), and a 'free' component of magnitude

$$
e_{1, \text { free }}=\left(e_{10}^{2}+e_{1 \mathrm{f}}^{2}-2 e_{1 \mathrm{f}} k_{10}\right)^{1 / 2}
$$

which rotates with frequency $g$. Note that $e_{1 \mathrm{f}}$ is independent of the mass of the outer planet, but the frequency $g$ is proportional to that mass.

Apsidal libration occurs if $e_{1, \text { free }} \leq e_{1 \mathrm{f}}$. In the $\left(k_{10}, h_{10}\right)$ plane, this condition is satisfied in the area of intersection of two circles, one of radius $e_{10}$ centered at the origin, the other of radius $e_{1 \mathrm{f}}$ centered at $\left(e_{1 \mathrm{f}}, 0\right)$, as shown in Fig. $2 \mathrm{~b}$. Assuming random values of $\varpi_{10}$ and $e_{10} \leq \varepsilon$, the probability $P_{\text {lib }}$ that the impulse on the outer planet will result in apsidal libration is simply the intersection area as a fraction of the area of the circle of radius $\varepsilon$. This is readily evaluated:

$$
P_{\mathrm{lib}}\left(e_{10} \leq \varepsilon\right)=\frac{1}{\pi}\left[\cos ^{-1}\left(\frac{\varepsilon}{2 e_{1 \mathrm{f}}}\right)+2\left(\frac{e_{1 \mathrm{f}}}{\varepsilon}\right)^{2} \sin ^{-1}\left(\frac{\varepsilon}{2 e_{1 \mathrm{f}}}\right)-\frac{e_{1 \mathrm{f}}}{\varepsilon} \sqrt{1-\left(\frac{\varepsilon}{2 e_{1 \mathrm{f}}}\right)^{2}}\right] .
$$

For $\varepsilon \ll e_{2 \mathrm{f}}$, we have $P_{\text {lib }}=0.5$ : nearly all values of $\varpi_{10}$ between $-90^{\circ}$ and $+90^{\circ}$ result in apsidal resonance. For $\varepsilon=e_{1 f}$, the probability of apsidal resonance drops only slightly, to $\sim 0.4$.

For the general case, $m_{1} \neq 0$, the probability of apsidal resonance can be determined numerically. It is easy to see from the general solution (eq. 4) that $P_{\text {lib }}$ has only a weak dependence on the mass ratio $\mu=m_{1} / m_{2}$, and $P_{\text {lib }}$ remains close to 0.5 .

When apsidal libration occurs, the libration amplitude is a steep function of $\varpi_{10}$ and $e_{10} / e_{2 \mathrm{f}}$. This is illustrated in Fig. 3, for one choice of parameters, $\mu=0.5$ and $\alpha=0.33$, corresponding to the case of planets $\mathrm{C}$ and $\mathrm{D}$ of $v$ Andromedae.

\section{Discussion}

We have shown that in a two planet system, an impulse perturbation on the eccentricity of the outer planet can excite the eccentricity of the inner planet on a secular timescale, and will result in apsidal libration with a $\sim 50 \%$ probability. There are two significant characteristics of the secular dynamics following an eccentricity impulse, whether or not it results in apsidal resonance: (i) the inner planet's eccentricity has large amplitude variation, and it drops to its initial small value periodically; (ii) only a small fraction of time is spent at large values of $|\Delta \varpi|$ and small values of $e_{1}$ (see Fig. 1). Therefore, observing small values of $|\Delta \varpi|$ and large values of $e_{1}$ is not surprising if 
the system has suffered an impulse perturbation in its history and the secular dynamics indicates large amplitude variations.

We note that in the opposite limit of a slow adiabatic perturbation which increases one planet's eccentricity on a timescale much longer than the secular timescale, apsidal resonance will occur with nearly 100\% probability and the resulting libration amplitude will be very small (for initially circular orbits). It is rather remarkable that the secular dynamics of two planets admits a high probability of apsidal resonance in both the impulse and the adiabatic limit of eccentricity perturbation of one planet. We defer detailed analysis to a future study, but we note here that the apsidal libration amplitude provides an observational diagnostic: large amplitudes favor the impulse mechanism whereas small amplitudes favor the adiabatic mechanism.

One possible mechanism for the eccentricity impulse is planet-planet scattering. Highly eccentric orbits can be produced by gravitational interactions of planets initially on circular orbits that are close to the threshold of orbital instability. This has been proposed as a possible explanation for the eccentric orbits of exo-planets (Rasio \& Ford 1996, Weidenschilling \& Marzari 1996, Lin \& Ida 1997, Ford et al. 2001, Marzari \& Weidenschilling 2002). There are three possible outcomes of such interactions: a final stable configuration different from initial, the merger of two planets, or the ejection of one planet. Numerical experiments thus far have explored only a small fraction of the parameter space of two and three giant-planet systems. In systems of two equal mass planets, comparable branching ratios for the three possible outcomes are found; the two former outcomes do not result in eccentricity excitation, but the third - ejection of a planet - does, with typical eccentricity of the surviving planet in the range $0.4-0.8$ (Ford et al. 2001). One problematic aspect is that two planet systems must have initial orbits fine-tuned close to the threshold of dynamical instability, i.e. orbital separation close to $2 \sqrt{3}$ times their mutual Hill radius (Gladman 1993). But this problem does not exist in systems of three or more planets, where chaotic instabilities can occur over a wider range of orbital separations; such systems can persist in quasi-stable orbital configurations for long periods of time, possibly exceeding $10^{8}$ years, before becoming dynamically unstable; then the most common outcome is the ejection of one planet (Marzari \& Weidenschilling 2002). Also, in the more realistic case of unequal masses, the probability of planet ejection is likely to be enhanced further.

We consider the ejection into an unbound orbit of a third planet by gravitational scattering from the outer of the two planets whose secular dynamics we have analyzed in section 2. (For comparable masses, ejection by scattering from the outer of the two planets is more likely than from the inner planet because the escape velocity is smaller at larger distance from the star: $v_{\mathrm{esc}} \sim r^{-1 / 2}$; it is also likely to produce the least direct perturbation to the inner members of the system.) Assuming that the ejected planet leaves on a nearly parabolic orbit, energy and angular momentum conservation yield the following estimate for the final eccentricity of the bound planet:

$$
1-e_{2 \mathrm{f}}^{2} \simeq\left(1+\frac{m_{3}}{m_{2}} \frac{a_{2}}{a_{3}}\right)\left[1+\frac{m_{3}}{m_{2}} \sqrt{\frac{m_{\star}+m_{2}}{m_{\star}+m_{3}}}\left(\sqrt{\frac{a_{3}}{a_{2}}}-\sqrt{\frac{2 q_{3}}{a_{2}}}\right)\right]^{2},
$$

where $m_{3}, a_{3}, q_{3}$ are the mass, initial semimajor axis and final periastron distance of the ejected 
planet; $a_{2}$ is the initial semimajor axis of $m_{2}$, and is related to its final semimajor axis by energy conservation: $a_{2 \mathrm{f}} \approx m_{2} a_{2} a_{3} /\left(m_{2} a_{3}+m_{3} a_{2}\right)$. Unfortunately, there is no additional dynamical constraint or simple argument to constrain the final periastron distance of the ejected planet. So the final eccentricity of the bound planet cannot be predicted analytically and must be determined numerically. Still, we can make the following estimate: since the periastron distance of the ejected planet is likely to be not greatly in excess of the apoastron distance of the surviving planet, then, for $q_{3} / a_{2} \simeq 1-1.5$, the final eccentricity of the bound planet will be in the range of $0.2-0.7$ for $m_{3} / m_{2} \simeq 0.1-0.7$.

Once a dynamical instability sets in, the subsequent evolution is highly chaotic and unpredictable in detail. A simplified description, based upon the presently available numerical simulations, is as follows: at first, close encounters between $m_{2}$ and $m_{3}$ lead to a rapid increase in their orbital eccentricities, over $\sim 10-10^{2}$ orbital periods; this is followed by a longer period, $\gtrsim 10^{4}$ orbits, during which many weak distant encounters gradually and stochastically increase the orbital period and apoastron of $m_{3}$, the planet-to-be-ejected. In the present context, the initial eccentricity excitation of the surviving planet, $m_{2}$, satisfies the impulse approximation as the timescale of the first stage is much shorter than the secular timescale (which is of order $\left(m_{1,2} / m_{\star}\right)^{-1}$ times the orbital period, or $\sim 10^{3}$ orbital periods for jovian mass planets). Thus, the secular dynamics of the two surviving planets, $m_{1}$ and $m_{2}$, would be as described in Section 2. The weak distant encounters that eventually lead to the ejection of the third planet would produce small perturbations to the secular solution, as would the gravitational interactions between $m_{1}$ and $m_{3}$. (Neglecting the latter is justified, in the lowest order, as the $m_{1}, m_{3}$ separation is much greater than the $m_{2}, m_{3}$ separation.) The effects of these perturbations on the secular dynamics presented above will be considered in a future study.

The case of $v$ Andromedae The analysis given in the previous section can be used to estimate the pre-impulse orbital eccentricity of at least one of the planets. A good candidate amongst the known exo-planetary systems to apply this theory is the $v$ Andromedae system, which represents perhaps the most (dynamically) constrained of the known exo-planetary systems. A recent orbital solution for this system (as quoted in Chiang et al. 2001) gives present eccentricities of planets C and D of 0.25 and 0.34 , respectively, and yields secular dynamics for these two planets similar to that shown in Fig. 1(e,f), with apsidal libration amplitude of $25^{\circ}-35^{\circ}$ (for relative orbital inclinations $\lesssim 20^{\circ}$ ). An independent orbital solution obtained previously by Stepinski et al. 2000 (from an older and therefore smaller set of observational data) has orbital parameters within $\sim 2 \sigma$ uncertainties of the more recent solution; it yields secular dynamics similar to that shown in Fig. 1(a,b), with apsidal libration amplitude of $80^{\circ}-90^{\circ}$. The libration period for this system is $\sim 7 \times 10^{3} \mathrm{yr}$. Now, if we allow that planet $\mathrm{D}$ suffered an eccentricity impulse, $e_{D} \approx 0.35$, in its history, we can estimate that the initial eccentricity of planet $\mathrm{C}$ could have been as small as $\sim 0.07$ if the apsidal libration amplitude is 25-35 deg, but even smaller, 0-0.01, if the amplitude is $80-90^{\circ}$.

After submitting this paper, we became aware of a recent preprint by Chiang \& Murray (2002) who propose an adiabatic eccentricity perturbation to explain the apsidal resonance in the 
$v$ Andromedae planetary system. They invoke torques from an exterior massive primordial disk to provide the adiabatic eccentricity excitation to the outermost planet D. However the adiabaticity of the perturbation is owed to a specific choice of disk parameters, which are not well constrained; a different, and perhaps equally plausible, choice of disk parameters may instead provide an impulse perturbation (E. Chiang 2002, personal communication); then the secular dynamics described here would apply.

As mentioned previously, the apsidal libration amplitude provides an observational diagnostic: large amplitudes favor the impulse mechanism whereas small amplitudes favor the adiabatic mechanism. In the case of $v$ Andromedae, there remain significant uncertainties in the orbital parameters, and at present it appears difficult to discriminate between the adiabatic and the impulse mechanisms. The 25-35 deg apsidal libration amplitude found by Chiang et al. (2001) is neither clearly small nor clearly large; in both the adiabatic mechanism and the impulse mechanism, an initial eccentricity near $0.06-0.07$ of planet $\mathrm{C}$ can account for this libration amplitude. On the other hand, the large libration amplitude of 80-90 deg found by Stepinski et al. (2000) would be explained by initial $e_{C} \lesssim 0.01$ in the impulse limit, but cannot be explained naturally in the adiabatic limit. We urge further observations and analysis to improve the accuracy and fidelity of orbital solutions of exo-planetary systems; this would help to constrain their dynamical history.

We acknowledge comments from E. Chiang, discussions with F. Rasio, and research support from NASA.

\section{REFERENCES}

Chiang, E.I., Tabachnik, S., \& Tremaine, S. 2001, AJ, 122, 1607

Chiang, E.I., \& Murray, N. 2002, ApJ, in press

Ford, E.B., Havlickova, M., \& Rasio, F.A. 2001, Icarus, 150, 303

Gladman, B. 1993, Icarus 106, 247

Lin, D.N.C. \& Ida, S. 1997, ApJ, 447, 781

Marzari, F., \& Weidenschilling, S.J. 2002, Icarus, 156, 570

Murray, C.D. \& Dermott, S.F. 1999, Solar System Dynamics (New York:Cambridge University Press)

Rasio, F. \& Ford, E. 1996, Science, 274, 954

Stepinski, T.F., Malhotra, R. \& Black, D.C. 2000, ApJ, 545, 1044

Weidenschilling, S.J. \& Marzari, F. 1996, Nature, 384, 619 
Wu, Y. \& Goldreich, P. 2002, ApJ, 564, 1024 

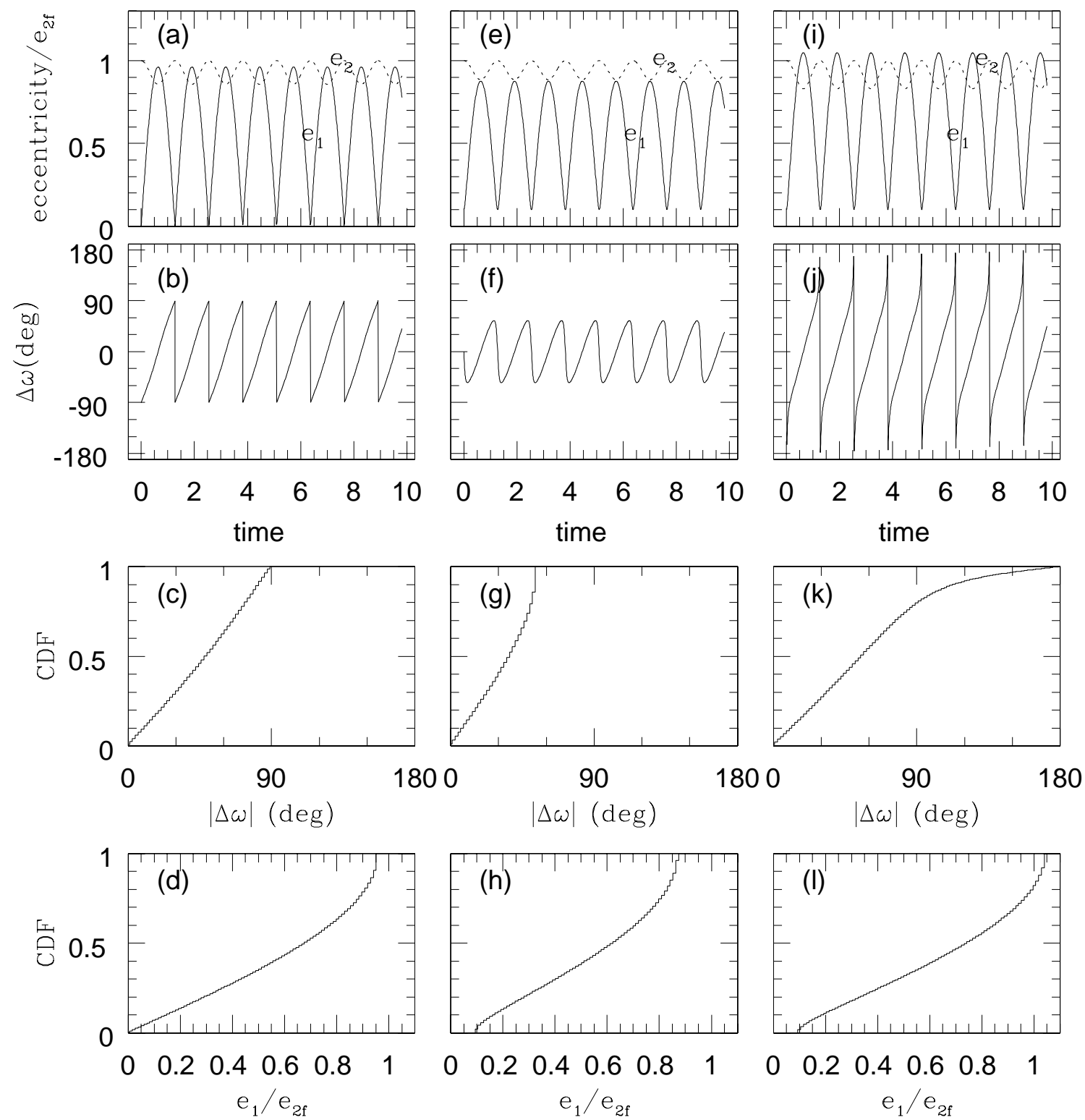

Fig. 1. - Secular dynamics for $\mu=0.5, \alpha=0.33$ and the following initial conditions: (a) - (c) $e_{10}=0, \varpi_{10}=0$; (d)-(f) $e_{10}=0.1 e_{2 \mathrm{f}}, \varpi_{10}=0$; (g)-(i) $e_{10}=0.1 e_{2 \mathrm{f}}, \varpi_{10}=\pi$. The upper two panels show the time variation of the eccentricities $e_{1}, e_{2}$, and the difference of apsidal longitudes, $\Delta \varpi=\varpi_{1}-\varpi_{2}$; the unit of time is $2 \pi / g_{1}$. The lower two panels plot the cumulative probability density function of $|\Delta \varpi|$ and of $e_{1}$. 

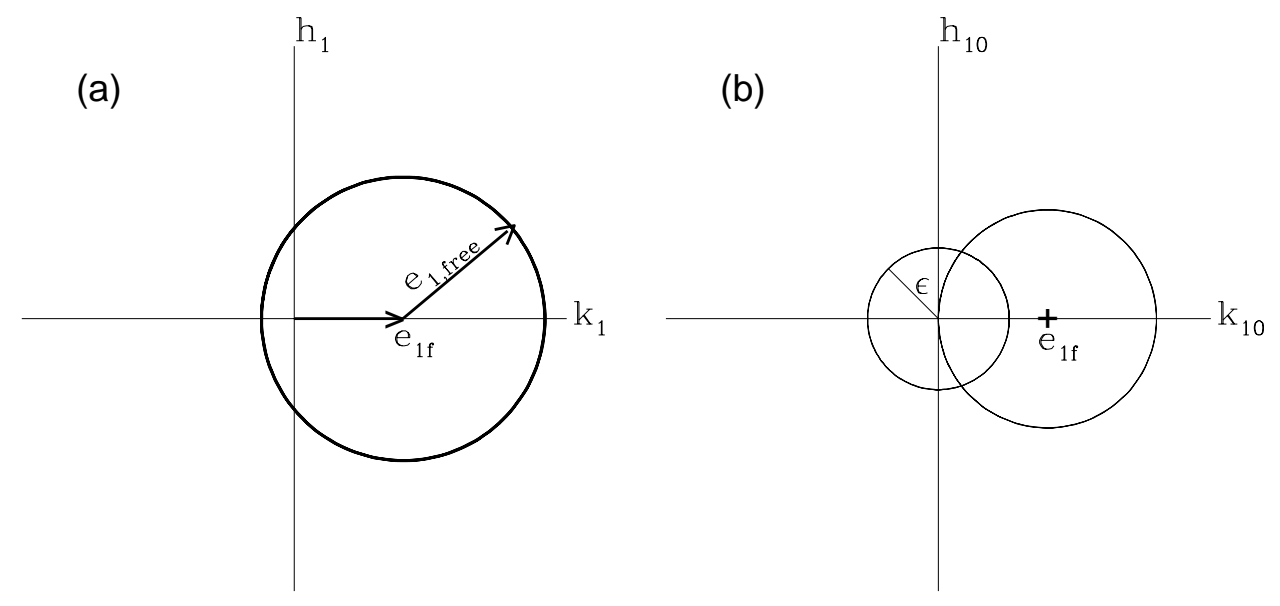

Fig. 2.- For $m_{1}=0$, the secular variation of $m_{1}$ 's eccentricity vector is the vector sum of a forced component of magnitude $e_{1 \mathrm{f}}$ along the direction of $m_{2}$ 's apsidal longitude and a rotating free component of magnitude $e_{1 \text {,free }}$ (a). For initial $e_{10}<\varepsilon$, apsidal resonance occurs for initial conditions $\left(k_{10}, h_{10}\right)$ in the area of intersection of the two circles shown in (b). 


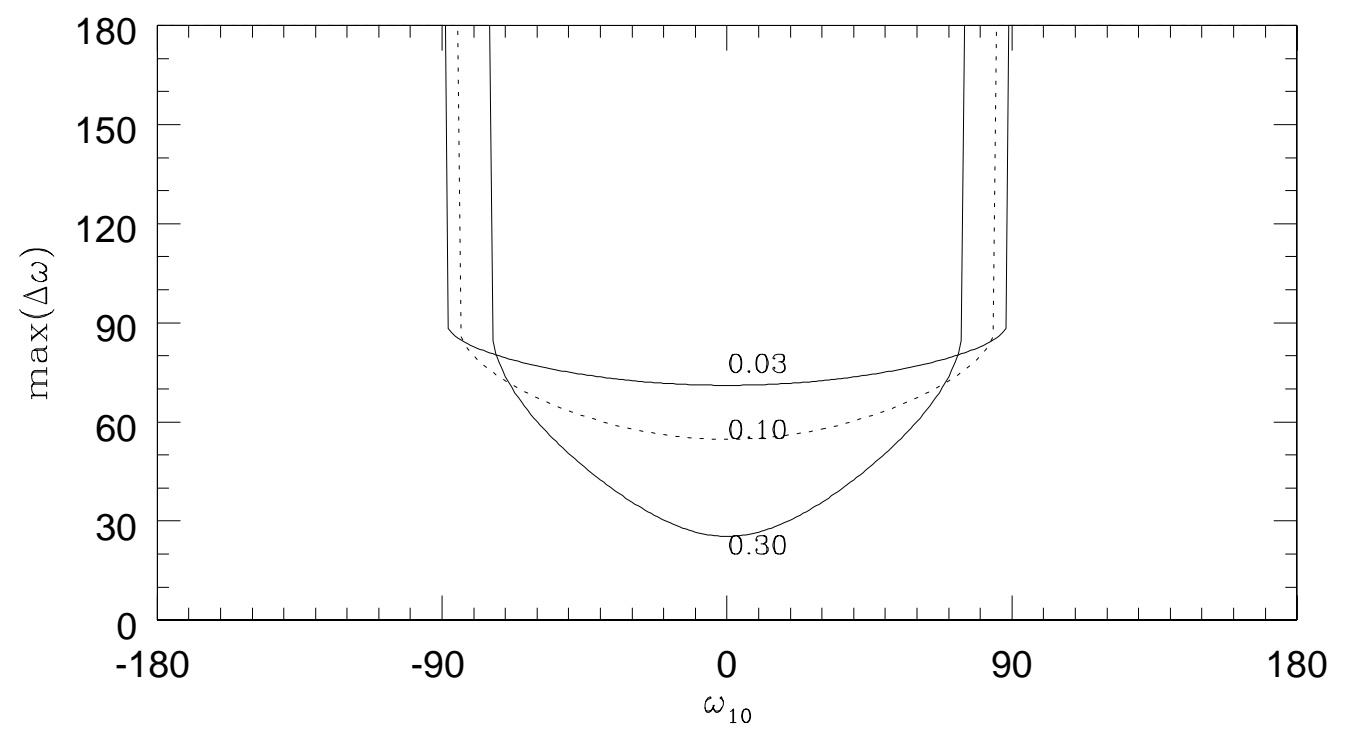

Fig. 3.- Apsidal libration amplitude as a function of initial $\varpi_{10}$ for $\mu=0.5, \alpha=0.33$, and three different values of $e_{10} / e_{2 \mathrm{f}}=0.03,0.1,0.3$. 